\title{
Perfil de óbitos por Covid-19 no Estado do Paraná no início da pandemia: estudo transversal
}

\author{
Profile of deaths by Covid-19 in the State of Paraná \\ at the beginning of the pandemic: a cross-section \\ study
}

Vanessa Cristine Ribeiro Fredrich ${ }^{1}$, Acácia Maria Lourenço Francisco Nasr ${ }^{2}$, Luciana Champion $^{3}$, Thiago Picussa de Campos Mello ${ }^{4}$, João Vittor Almeida da Silva ${ }^{5}$, Mauren Letícia Zialk ${ }^{6}$, Alexandre Mario Bellizzi ${ }^{7}$, Maria Goretti David Lopes ${ }^{8}$, Nestor Werner Junior ${ }^{9}$, Carlos Alberto Gebrim Preto ${ }^{10}$

1. ORCID: https://orcid.org/0000-0002-2571-5358. Médica. Especialista em Clínica Médica. SESA, Curitiba, Paraná, Brasil. E-mail: vanessa.fredrich@sesa.pr.gov.br.

2. ORCID: https://orcid.org/0000-0002-7663-6094. Médica. Coordenadora da Vigilância em Saúde. SESA, Curitiba, Paraná, Brasil. E-mail: acacianasr@gmail.com.

3. ORCID: https://orcid.org/0000-0002-8436-0358. Médica. Medicina Intensiva Pediátrica. SESA, Curitiba, Paraná, Brasil. E-mail: luciana.champion@sesa.pr.gov.br.

4. ORCID: https://orcid.org/0000-0003-0611-4044 Acadêmico de Medicina. UFPR, Curitiba, Paraná, Brasil. E-mail: thiagopicussa@gmail.com.

5. ORCID: https://orcid.org/0000-0003-4001-0636 Acadêmico de Informática Biomédica. Estagiário nível superior. SESA, Curitiba, Paraná, Brasil. E-mail: est.joaosilva@sesa.pr.gov.br.

6. ORCID: https://orcid.org/0000-0002-8890-5669 Médica. Mestra em Saúde Coletiva. Faculdades Pequeno Príncipe. Curitiba, Paraná, Brasil. E-mail: maurenziak@gmail.com.

7. ORCID: https://orcid.org/0000-0002-7101-136X Enfermeiro. Chefe Divisão de Vigilância de Informações Epidemiológicas. SESA, Curitiba, Paraná, Brasil. E-mail: alexandre.bellizzi@sesa. pr.gov.br.

8. ORCID: https://orcid.org/0000-0001-7473-6342 Enfermeira. Diretora de Atenção e Vigilância em Saúde. SESA, Curitiba, Paraná, Brasil. E-mail: maria.lopes@sesa.pr.gov.br.

9. ORCID: https://orcid.org/0000-0003-4257-1752 Dentista. Diretor Geral. SESA, Curitiba, Paraná, Brasil. E-mail: nestor.jr@sesa.pr.gov.br.

10. ORCID: https://orcid.org/0000-0002-7132-7372 Médico. Secretário de Estado da Saúde do Paraná. SESA, Curitiba, Paraná, Brasil. E-mail: betopreto@sesa.pr.gov.br.

CONTATO: Nome do autor correspondente: Vanessa Cristine Ribeiro Fredrich | Endereço: Rua Piquiri, 170. Curitiba, Paraná. CEP 80230-140 | Telefone: 4133304751 | E-mail: vanessa.fredrich@sesa.pr.gov.br 
RESUMO O Novo Coronavírus representa uma emergência em saúde pública, trazendo desafios à vigilância epidemiológica. As estatísticas de mortalidade são primordiais nesse contexto e registros condizentes com a realidade dependem de um trabalho coordenado entre os serviços e técnicos que trabalham com os sistemas de informação em saúde. O objetivo foi descrever o papel da vigilância epidemiológica na identificação do perfil dos óbitos por Covid-19 no início da pandemia no Estado do Paraná. Trata-se de um estudo transversal a partir de dados do SIM - Sistema de Informação em Mortalidade, dados preliminares até a semana epidemiológica 22 (25 de maio de 2020). O perfil de óbitos por Covid-19 no Estado do Paraná com dados até maio de 2020 mostrou características semelhantes às de outras localidades, com maior acometimento do sexo masculino (68\%), idosos (67\%) e portadores de condições prévias (69\%), sendo as principais delas hipertensão (23\%), diabetes (21\%) e cardiopatia (13\%). A equipe de vigilância epidemiológica dos municípios e regionais de saúde tem papel fundamental na captação e qualificação dos dados de mortalidade e, no contexto pandêmico, se beneficia de orientações oportunas e comunicação eficiente por parte do nível estadual de gestão.

DESCRITORES: Covid-19. Vigilância em Saúde Pública. Estatísticas Vitais.

\begin{abstract}
The New Coronavirus represents a public health emergency, bringing challenges to health surveillance such as the need to establish information flows in order to produce reliable statistics to support management actions. Statistics on mortality are essential in this process and records consistent with reality depend on coordinated work between services and information systems. The objective has to describe the role of epidemiological surveillance in identifying the profile of deaths by Covid-19 at the beginning of the pandemic in the State of Paraná. Cross-sectional study using data from SIM - Mortality Information System, preliminary data until epidemiological week 22 (May 27, 2020). The mortality profile of deaths by Covid-19 in the state of Paraná with data up to May 2020 showed similar characteristics to the profiles of other locations, demonstrating to affect mostly males (68\%), older population (67\%) and people with previous conditions (69\%), with the main comorbidities being hypertension (23\%), diabetes (21\%) and heart disease (13\%). The epidemiological surveillance team of municipalities and regions of health has a fundamental role in identifying and qualifying mortality data and, in the pandemic context, benefits from timely guidance and efficient communication by the state level of management.
\end{abstract}

DESCRIPTORS: Covid-19. Public Health Surveillance. Vital statistics. 


\section{INTRODUÇÃO}

m 30 de janeiro de 2020, a Organização Mundial de Saúde (OMS) declarou - o surto da doença pelo Novo Coronavírus como uma emergência em saúde pública, e em 11 de março de 2020 como pandemia. Até a data de 24 de maio de 2020 a OMS havia reportado o número de 5.204 .508 casos em todo o

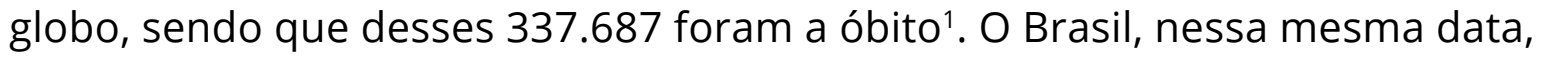
alcançou a marca de 347.398 casos e 22.013 mortes, com uma dispersão heterogênea ao longo de todo o território nacional. O Estado do Paraná notificou o primeiro caso de Covid-19 em 17 de março de 2020 e teve o primeiro óbito registrado 10 dias após. Considerando as diferentes políticas de testagem entre os estados, países e municípios, percebe-se o desafio de se aproximar da real situação epidemiológica e compará-las, já que a maioria dos locais realiza os testes para o Coronavírus apenas em pacientes sintomáticos, principalmente nos que apresentam sintomas mais graves. Desta forma, estima-se que ocorra subnotificação dos casos totais na ordem de sete a oito vezes².

No atual cenário, as estatísticas de mortalidade são primordiais ao acompanhamento da evolução da epidemia, e para que isso se efetive os registros devem ser precisos e oportunos. Assim, a declaração de óbito (DO) é o documento padrão para a coleta das informações sobre mortalidade, servindo de base para o cálculo das estatísticas vitais e epidemiológicas do país 3 . As fontes de informação para monitoramento dos óbitos pela Secretaria Estadual de Saúde (SESA-PR) são a planilha de monitoramento diária do Centro de Informações Estratégicas em Vigilância em Saúde (CIEVS), o sistema de notificação de casos suspeitos de Covid-19 do Paraná - "Notifica Covid-19", o Sistema de Informação em Mortalidade (SIM), Sistema de Informação da Vigilância Epidemiológica da Gripe (SIVEP), Laboratório Central do Estado (LACEN), Sistema de Notificação do Ministério da Saúde (e-SUS VE), portal da transparência de registro civil, entre outras.

A obtenção de dados fidedignos depende não somente de um registro ade- 
quado por parte dos profissionais de saúde em cada um desses sistemas, como também de uma equipe dedicada à qualificação dos dados e à sua interpretação à luz de fatores limitantes e/ou de confusão. A qualificação, organização e divulgação desses dados na forma de boletins epidemiológicos são imprescindíveis para a tomada de decisões tanto pela equipe de saúde na linha de frente quanto para a alocação de recursos por parte da gestão. Diante disso, este estudo tem por objetivo descrever o papel da vigilância epidemiológica na identificação do perfil e distribuição dos óbitos por Covid-19 no início da pandemia no Estado do Paraná.

\section{MÉTODO}

Estudo descritivo e transversal dos óbitos confirmados para Covid-19 no Estado do Paraná, registrados no SIM, utilizando os dados parciais disponíveis até 25 de maio de 2020. O estudo foi conduzido na Coordenadoria de Vigilância Epidemiológica da Secretaria de Estado da Saúde. A fonte de consulta foram as planilhas em formato csv disponibilizadas em página da internet da SESA-PR ${ }^{4}$ no dia 27 de maio, com dados do SIM do dia 25 de maio de 2020, além dos próprios boletins epidemiológicos disponíveis na referida página. Optou-se por utilizar os dados públicos já divulgados pela SESA-PR, os quais tiveram como fonte o SIM, ao invés dos contidos diretamente no sistema de mortalidade, uma vez que os dados públicos já passaram por qualificação, ou seja, incluem apenas casos confirmados. Por se tratar de dados obtidos a partir de planilhas de acesso livre na página da SESA-PR, justifica-se a dispensa de aprovação por comitê de ética em pesquisa.

As taxas de incidência, mortalidade e letalidade por macrorregião de residência utilizaram os dados divulgados no boletim epidemiológico estadual do dia 18 de maio; já as variáveis faixa etária, sexo, raça/cor, escolaridade, descrição da ocupação e registro de condições clínicas associadas na DO são do banco de dados disponibilizado com dados do SIM do dia 25 de maio de 2020. 
Os dados secundários foram coletados a partir do banco de dados estadual e estão em acordo com a Resolução 466 de 2012 (CNS, 2012) $)^{5}$, que trata sobre as diretrizes e normas regulamentadoras de pesquisa envolvendo seres humanos, tendo sido dispensado o Termo de Consentimento Livre e Esclarecido. Os dados foram tabulados em planilha do programa Microsoft Office Excel ${ }^{\circledR}$ versão 2007.

No boletim epidemiológico divulgado no dia 27 de maio, constatou-se 162 óbitos em residentes do Paraná, contra 104 registros encontrados no SIM (dados tabulados em 25 de maio), ou seja, coincidência de 64\%. Essa diferença é esperada, uma vez que o total de casos e óbitos divulgados em boletim tem como fonte de informação a planilha de monitoramento do CIEVS e a planilha usada para perfil de óbitos teve como fonte o SIM. Segundo o Ministério da Saúde, a suspeita de Covid-19 deve ser registrada pelos médicos na declaração de óbito e digitada nos municípios em até 48 horas $^{6}$. Após essa digitação preliminar, os dados passam por investigação e qualificação com alterações subsequentes o que pode levar de alguns dias a semanas para ocorrer, justificando o atraso nos dados nesse sistema. Importante salientar que todos os 104 registros de declarações de óbitos incluídos tiveram preenchimento dos campos sexo, idade, raça/cor e ocupação. A escolaridade teve $85 \%$ de completude.

\section{RESULTADOS}

A Tabela 1 apresenta as características dos óbitos por Covid-19 em residentes do Paraná de acordo com idade, sexo, raça/cor e escolaridade.

Tabela 1. Perfil dos óbitos por Covid-19 em residentes do Paraná segundo idade, sexo, raça/ cor, escolaridade e presença de condições associadas até 25 de maio de 2020. 
Óbitos - $\mathrm{n}^{\circ}$

Faixa etária (anos)

Média

$<60$ anos $(\%)$

$\geq 60$ anos (\%)

Sexo - $n^{\circ}(\%)$

Masculino

Feminino

Cor $-n^{\circ}(\%)$

Branca

Negra

Amarela

Escolaridade $-n^{\circ}(\%)$

Fundamental I

Fundamental II

Ensino médio

Superior incompleto

Superior completo

Condição clínica associada - $n^{\circ}(\%)$

Presente

Ausente
104

67 (17 - 95 anos)

34 (33)

$70(67)$

71 (68)

33 (32)

$83(80)$

18 (17)

3 (3)

$6(7)$

7 (8)

27 (30)

24 (26)

26 (29)

75 (69)

34 (31)

Fonte: Elaborado pelos autores (2020). Elaboração própria a partir de arquivos csv com dados do SIM divulgados na página da SESA-PR do dia 25/05/2020.

Quase 70\% dos óbitos tiveram condições clínicas associadas à Covid-19 registradas em alguma linha da declaração de óbito (Tabela 2). 
Tabela 2. Lista de condições clínicas associadas informadas na declaração de óbito por Covid-19 no Estado do Paraná.

\begin{tabular}{llc}
\hline Condição Clínica* & $\mathbf{n}^{\mathbf{0}}$ & $\%$ \\
\hline Hipertensão arterial sistêmica & 26 & 23.6 \\
Diabetes mellitus & 24 & 21.8 \\
Cardiopatia & 15 & 13.6 \\
Doença renal crônica & 10 & 9.1 \\
Obesidade & 9 & 8.2 \\
Doença pulmonar & 8 & 7.3 \\
Neoplasia & 6 & 5.5 \\
Acidente vascular cerebral & 4 & 3.6 \\
Demência & 2 & 1.8 \\
Etilismo & 2 & 1.8 \\
Tabagismo & 1 & 0.9 \\
Outros & 3 & 2.7 \\
TOTAL & 110 & 100 \\
\hline
\end{tabular}

Fonte: Elaborado pelos autores (2020). Adaptação de informe epidemiológico do dia 27/05/2020. *uma mesma pessoa pode apresentar mais de uma condição clínica associada.

Quanto à ocupação, destacam-se óbitos em aposentados, trabalhadores da área administrativa, do lar, autônomos, comerciantes e motoristas. As informações sobre ocupação foram agregadas por áreas afins na Tabela 3. 
Tabela 3. Distribuição dos óbitos de Covid-19 do Estado do Paraná segundo ocupação.

\begin{tabular}{ll}
\hline Ocupação & $\mathbf{N}^{\circ}(\%)$ \\
\hline Aposentado & $20(18,3)$ \\
Administrativo & $18(16,5)$ \\
Do Lar & $13(11,9)$ \\
Autônomo & $11(10,1)$ \\
Comerciante & $9(8,3)$ \\
Motorista & $8(7,3)$ \\
Rural & $6(5,5)$ \\
Saúde & $5(4,6)$ \\
Indústria & $4(3,7)$ \\
Segurança & $3(2,8)$ \\
Engenheiro & $2(1,8)$ \\
Outros & $5(9,2)$ \\
TOTAL & $104(100)$ \\
\hline
\end{tabular}

Fonte: Elaborado pelos autores (2020). Elaboração própria a partir de arquivos csv com dados do SIM divulgados na página da SESA-PR do dia 25/05/2020.

A Tabela 4 apresenta a distribuição dos óbitos por Covid-19 segundo macrorregiões do Paraná. Ao se analisar a distribuição espacial desses óbitos no Estado, maiores taxas de mortalidade e letalidade foram observadas nas macrorregiões Norte e Noroeste, onde atingiram valores acima da média estadual. Na região Noroeste e Norte, foram observadas taxas de mortalidade de 1,6 e 1,5 casos por cem mil habitantes, respectivamente, enquanto a região Oeste, com a menor taxa de mortalidade do Estado, apresentou 0,6 óbitos por cem mil habitantes. Quanto à letalidade, que se refere ao número de óbitos dentre os pacientes infectados, a região Norte apresentou um número elevado, cerca de 10\%, enquanto a região Oeste apresentou a menor taxa paranaense, com 3,3\%. 
Tabela 4. Distribuição dos óbitos por Covid-19 segundo macrorregiões do Paraná.

\begin{tabular}{llllll} 
& Paraná & Leste & Norte & Noroeste & Oeste \\
\hline População* & 11433957 & 5586569 & 1988697 & 1879867 & 1978824 \\
Casos & 2360 & 1133 & 299 & 536 & 392 \\
$\begin{array}{l}\text { Óbitos } \\
\text { Taxa de mortalidade }\end{array}$ & 127 & 52 & 31 & 31 & 13 \\
(por 100 mil) & 1,1 & 0,9 & 1,5 & 1,6 & 0,6 \\
Taxa de letalidade - \% & 5,4 & 4,6 & 10,4 & 5,8 & 3,3 \\
Incidência (por 100 mil) & 20,6 & 20,2 & 15,0 & 28,5 & 19,8 \\
\hline
\end{tabular}

Fonte: Elaborado pelos autores (2020). Elaboração própria a partir de Informe Epidemiológico da Secretaria Estadual de Saúde do Paraná de 18/05/2020. *Segundo estimativas do IBGE para o ano de 2019.

Comparando os dados dos informes oficiais das Secretarias Estaduais de Saúde e do Ministério da Saúde apresentados na Tabela 5, observamos que a taxa de incidência da Covid-19 nos Estados do Paraná e Mato Grosso do Sul apresentou-se abaixo da média nacional, ao passo que os Estados do Rio de Janeiro e Amazonas apresentaram taxa de incidência acima da média nacional.

Tabela 5. Taxas de mortalidade, letalidade e incidência de Covid-19 em alguns Estados brasileiros.

\begin{tabular}{lccccc}
\hline & Brasil & RJ & AM & MS & PR \\
$\begin{array}{l}\text { Taxa de mortalidade } \\
\text { (por 100 mil) }\end{array}$ & 7,9 & 16,4 & 34,1 & 0,6 & 1,1 \\
$\begin{array}{l}\text { Taxa de letalidade \%. } \\
\begin{array}{l}\text { Incidência Covid-19 } \\
\text { (por 100 mil) }\end{array}\end{array}$ & $6,60 \%$ & $10,70 \%$ & $6,80 \%$ & $2,60 \%$ & $5,40 \%$ \\
\hline
\end{tabular}

Fonte: Elaborado pelos autores (2020). Elaboração própria a partir de dados do Ministério da Saúde e Secretarias Estaduais (informes do dia 18/05/2020)7-9. 


\section{DISCUSSÃO}

Dados divulgados pela SESA-PR na semana epidemiológica 22 revelaram média de idade dos casos confirmados para Covid-19 de 44 anos, com distribuição similar entre homens e mulheres. Já na análise do perfil dos pacientes que evoluíram para óbito, observamos maior mortalidade em idosos (67\% acima de 60 anos) do sexo masculino (67\%). Dado semelhante foi observado em estudo com população chinesa, no qual $64 \%$ eram homens, com média de idade de 70 anos e $84 \%$ acima de 60 anos. Ainda, esse estudo chinês demonstrou que cerca de $75 \%$ dos óbitos ocorreram em pessoas com condições prévias como hipertensão (41\%), diabetes (29\%), cardiopatia (27\%), doenças pulmonares $(23 \%)$ e cerebrovasculares $(12 \%)^{10}$. Na amostra paranaense, as condições associadas mais encontradas também foram hipertensão (23\%), diabetes (21\%) e cardiopatia (13\%). Sobre o excesso de mortalidade em homens, sabe-se que fatores sociais e culturais, como menor procura de serviços primários de atenção à saúde, interferem com a saúde dessa população, o que poderia justificar a maior mortalidade nesse grupo ${ }^{11}$.

No início da pandemia, estratégias de isolamento vertical foram cogitadas, as quais priorizam o isolamento de idosos mantendo as atividades dos demais grupos, tanto de trabalhadores quanto de escolares. Algumas críticas a esse modelo de enfrentamento incluíram a constatação de que crianças, apesar de apresentarem tendência a formas mais leves da doença, transmitiam a infecção a seus familiares ${ }^{12}$.

A predominância de óbitos entre aposentados e trabalhadores do lar está de acordo com o esperado, uma vez que a faixa etária mais idosa é a que acumula mais comorbidades, o que eleva a letalidade nesse grupo. Dentre os trabalhadores que foram a óbito, houve predomínio nas chamadas áreas essenciais de trabalho, ou seja, em trabalhadores da saúde, transporte, segurança, além de profissionais autônomos, os quais, por terem menor segurança na renda, têm maior dificuldade em seguir as recomendações de isolamento social. 
Em relação à raça/cor, através da agregação de autodeclarados pretos e pardos sob a classificação de raça negra, observamos óbito de $17 \%$ nessa população. No Brasil, de acordo com o boletim número 16 publicado em 18 de maio de 2020, 25,2\% do total de casos ocorreram em pretos e pardos, e essa população correspondeu a 54,8\% do total de óbitos, demonstrando uma discrepância entre incidência e mortalidade em decorrência da raça/cor. Importante notar que $51,3 \%$ dos casos registrados no país naquele momento não tiveram raça/ cor informada, ou seja, essa diferença poderia ser ainda maior. Ainda assim, já era possível perceber maior letalidade na população negra comparada à branca. Um estudo dos EUA reportou que $45 \%$ dos casos de Covid-19 ocorreram em brancos, em contraste com 33,1\% em negros, proporção muito maior que a composição de pessoas negras naquela população (13\%). Alguns fatores implicados nessa diferença vão desde maior prevalência de doenças de base como diabetes, hipertensão, obesidade e asma, as quais são fatores de risco para formas graves da Covid-19, até maior proporção de pessoas negras trabalhando em serviços essenciais ou que não permitem trabalho remoto ${ }^{13}$.

A análise dos dados desagregados por macrorregião de saúde permite reconhecer heterogeneidades entre as localidades, identificando os locais que necessitam de maior suporte e medidas adequadas ao momento epidemiológico. Como exemplo, citamos a macrorregião Norte que, no momento avaliado, apresentou letalidade maior que a média do Estado (10,3\%), contrastando com a macrorregião Oeste (3,3\%). Sabe-se que há uma relação entre número de casos testados e taxa de letalidade, visto que se há limitação de testes disponíveis, a tendência é priorizar a testagem de casos mais graves, incorrendo-se em um viés de seleção. Esse viés leva a um aumento da taxa de letalidade, uma vez que pacientes infectados com sintomas leves ou oligossintomáticos não participam do cálculo ${ }^{14}$. Em países com testagem em massa, como por exemplo a Coreia do Sul, a taxa de letalidade ficou entre 1-2\%. De acordo com estudo realizado pela USP, a taxa de letalidade esperada para a doença, tomando como base os dados da Coreia do Sul e corrigindo para as faixas etárias da população do Brasil, seria de $1,11 \%{ }^{15}$. No Paraná, observou-se taxa de letalidade de 5,24\% 
no período analisado, o que sinalizou para uma necessidade de ampliação da disponibilidade de testes.

A obtenção de dados de mortalidade aproximados da real situação da pandemia configura um desafio aos técnicos das Secretarias de Saúde, uma vez que sofre interferência de variáveis como a completude de dados nos sistemas de informação, o preenchimento adequado das declarações de óbito pelos médicos, disponibilidade de testes diagnósticos, sensibilidade e especificidade dos testes e possibilidade de falsos negativos. Esse estudo teve como principal objetivo apresentar o trabalho da vigilância epidemiológica na identificação dos óbitos por Covid-19 no Estado do Paraná, tendo como limitações o tempo para qualificação dos dados e a própria pandemia, que torna o cenário dinâmico com atualização constante do número de casos e óbitos.

\section{CONCLUSÃo}

Uma resposta oportuna e eficiente à emergência em saúde pública pela Covid-19 demanda ações coordenadas entre os diferentes setores e áreas do conhecimento. O trabalho da vigilância epidemiológica torna-se imprescindível na qualificação e análise das estatísticas de mortalidade, as quais embasam ações como instituição de restrições de circulação, alocação de recursos humanos e insumos, expansão da rede assistencial e atenção a populações vulneráveis com vistas à redução de danos e preservação da saúde da população.

\section{REFERÊNCIAS}

1. Organização Pan-americana de Saúde [Internet]. [local desconhecido: OPAS; 2020. [citado em 2020 Jun 24]. Disponível em: https://www.paho.org/bra/COVID-19.

2. Hallal PC, Barros AJD, Horta BL, Dellagostin OA, Hartwig FP, Pellanda LC, Struchiner CJ, Burattini MN, Silveira MF, Menezes AMB, Barros FC, Victora CG. Population-based survey of COVID-19 in Southern Brazil. [submitted for publication].

3. Ministério da Saúde (Brasil). ConsFedMedCentBrasClassif Doenças. 2009; A declaração de óbito: documento necessário e importante [Internet] 3. ed. Brasília: Ministério da Saúde; 2009. 
4. Paraná. Secretaria de Estado da Saúde. Informe Epidemiológico do COVID-19. Curitiba: SESA; 2020. Disponível em: https://www.saude.pr.gov.br/Pagina/Coronavirus-COVID-19.

5. Ministério da Saúde. Conselho Nacional de Saúde. Resolução no 466, de 12 de dezembro de 2012. Disponível em https:// bvsms.saude.gov.br/bvs/saudelegis/cns/2013/res0466_12_12_2012.html.

6. Ministério da Saúde. Orientações para codificação das causas de morte no contexto da COVID-19. Brasil: Ministério da Saúde; 2020. [citado em 30 Set 2020]. Disponível em: https://portalarquivos2.saude.gov.br/images/pdf/2020/April/29/NotaInformativa-declara----o-obito.pdf.

7. Secretaria de Saúde do Estado do Amazonas. Boletim COVID-19 [Internet]. SES-AM; 2020. [citado em 2020 Jul 17]. Disponível em: http://coronavirus.amazonas.am.gov.br/wp-content/uploads/2019/03/IG-Boletim-Covid-19-14.png.

8. Secretaria de Estado de Saúde do Mato Grosso do Sul. Boletim Coronavírus - COVID-19 [Internet]. SES-MS; 2020. [citado em 2020 Jul 17]. Disponível em: https://www.vs.saude.ms.gov.br/wp-content/uploads/2020/05/Boletim-Epidemiol\%C3\%B3gicoCOVID-19-2020.05.

9. Secretaria de Saúde do Estado do Rio de Janeiro. Novo coronavírus - Boletim diária (18/05/2020) [Internet]. SES-R]; 2020. [acesso em 2020 jul 17]. Disponível em: https://coronavirus.rj.gov.br/boletim/boletim-coronavirus-18-05-2-852-obitos-e-26665-caso.

10. Sun YJ, Feng YJ, Chen J, Li B, Luo ZC, Wang PX. Clinical features of fatalities in patients with COVID-19 [published online ahead of print, 2020 Jul 15]. DisasterMedPublic Health Prep.; 2020. 1-10. doi:10.1017/dmp.2020.235.

11. Coelho EBS, Schwarz E, Bolsoni CC, Conceição TB. Política nacional de atenção integral a saúde do homem. 2018. 66 p.

12. Cao Q, Chen YC, Chen CL, Chiu CH. SARS-CoV-2 infection in children: Transmission dynamics and clinical characteristics [Internet]. J Formos Med Assoc.; 2020. 119(3):670-3. Disponível em: https://doi.org/10.1016/j.jfma.2020.02.00913.

13. Dyer O. Covid-19: Black people and other minorities are hardest hit in US [Internet]. BM].; 2020. 369(April):m1483. Disponível em: http://dx.doi.org/doi:10.1136/bmj.m1483.

14. Dornels C, Souza F De, Paulo J, Paiva S De, Leal TC, Feitosa L. Evolução espaço temporal da letalidade por COVID-19 no Brasil, 2020. J BrasPneumol.; 2020. 46(4):56-8.

15. Covid-19 Brasil. Monitoramento e análises da situação do Coronavírus no Brasil. Estimativa de Casos de Covid-19 [Internet]. Ribeirão Preto: USP; 2020. [citado em 2020 Jun 26]. Disponível em: https://ciis.fmrp.usp.br/covid19-subnotificacao/.

RECEBIDO:26/07/2020

ACEITO: 08/10/2020 